ORIGINAL

\title{
Efficacy of essence oil supplementation to feeds on volatile fatty acid production
}

\section{La eficacia de los suplementos de aceite de esencia en la producción de ácidos grasos volátiles}

\author{
Ahmet Tekeli, ${ }^{1 *}$ Ph.D, Gültekin Yıldız, ${ }^{2}$ Ph.D, Winfried Drochner, ${ }^{3}$ Ph.D, \\ Herbert Steingass, ${ }^{3}$ Ph.D.
}

\begin{abstract}
${ }^{1}$ Yuzuncu YII University, Faculty of Agriculture, Department of Animal Science, 65080, Van/Turkey. ${ }^{2}$ Ankara University, Faculty of Veterinary, Department of Animal Nutrition and Nutritional Diseases, 06110 Ankara/Turkey. ${ }^{3}$ Hohenheim University, Department of Animal Nutrition, 70599 Stuttgart/ Germany. Corresponding: atekeli@yyu.edu.tr
\end{abstract}

Received: October 2014; Acepted: March 2015.

\begin{abstract}
Objective. Determine the effect of some plant extract supplementation to Total Mixed Ration (TMR), concentrate and hay on volatile fatty acid (VFA) production at 8 and 24 hours (h) using in vitro gas production technique in cattle. Material and methods. Three fistulated Holstein dairy cows were used for rumen fluid collection for application of in vitro gas production technique. Four essence oils ( $T$. vulgaris, $O$. vulgare, $S$. aromaticum, $Z$. officinale) were used as plant extracts. Results. Essence oil supplementations to the examined feed groups had significant effect only on C2/C3 VFA level at $8 \mathrm{~h}$ in all feed groups $(\mathrm{p}<0.05)$. C2/C3 VFA level at $8 \mathrm{~h}$ significantly increased in the groups with Oregano $25 \mathrm{ppm}$ supplementation for TMR and concentrate and in the groups with Thymol $25 \mathrm{ppm}$ supplementation for hay. C3 VFA level at $8 \mathrm{~h}$ significantly increased in the group that received Syzygium $200 \mathrm{ppm}$ supplementation for hay. Different plant extracts supplemented to TMR, concentrate and hay significantly affected C2, C3, IC4, IC5, C5 and C2/C3 VFA levels at $24 \mathrm{~h}(\mathrm{p}<0.05)$. Conclusions. The findings of the study indicate that moderate doses of plant extracts result in increased VFA levels in ruminants while higher doses demonstrate the opposite effect.
\end{abstract}

Key words: Aromatic plants, in vitro, plant extract, ruminant nutrition, volatile fatty acid (Source: USDA).

\section{RESUMEN}

Objetive. Determinar el efecto de los suplementos de algunos extractos de plantas a Ración Total Mezclada (TMR), concentrado y heno sobre los ácidos grasos volátiles (AGV) a las 8 y 24 horas (h) utilizando la técnica de producción de gas in vitro en el ganado bovino. Material y métodos. Tres vacas lecheras Holstein fistuladas se utilizaron para la recogida de fluido ruminal y aplicar la técnica in vitro de producción de gas. Cuatro aceites esenciales (T. vulgaris, O. vulgare, S. aromaticum, Z. officinale) fueron utilizadas como extractos de plantas. Resultados. La suplementación de aceite esenciales a los grupos de alimentos estudiados tuvieron efecto significativo sólo en C2 / nivel C3 VFA a las 8 h en todos los grupos de alimentos $(\mathrm{p}<0.05)$. El C2 / nivel C3 VFA a las $8 \mathrm{~h}$ aumentó significativamente en los grupos con suplementación de orégano $25 \mathrm{ppm}$ de la RTM y concentrado y en los grupos con suplementación de Timol a 25 ppm para el heno. El C3 nivel de VFA en 8 h aumentó significativamente 
en el grupo que recibió suplementación de Syzygium a razón de 200 ppm para el heno. Los diferentes extractos de plantas suplementadas con RTM, concentrado y heno afectó significativamente los niveles de C2, C3, IC4, IC5, C5 y C2/C3 VFA a las $24 \mathrm{~h}(\mathrm{p}<0.05)$. Conclusiones. Los hallasgos del estudio indican que las dosis moderadas de extractos de plantas incrementan los niveles de AGV en rumiantes, mientras que dosis más altas producen el efecto contrario.

Palabras clave: Ácidos grasos volátiles, in vitro, extracto de la planta, nutrición de rumiantes, plantas aromáticas (Fuente: USDA).

\section{INTRODUCTION}

Energy and protein losses in rumen fermentation reduce the efficiency of nutrient utilization and cause environmental pollution. Ionophore antibiotics have been successfully used in rumens for many years to reduce energy and protein losses (1). While ionophore antibiotics have been widely used in conventional production systems for the control of rumen fermentation, the European Commission (EC) decided to phase out, and ultimately ban the use of antibiotics (January 1st 2006) (2). After the ban on antibiotic growth promoters, studies have increased on exploring alternatives to antibiotics. One of these possible alternatives are the plant extracts, which are also called as essence oils. Essence oils are plant secondary metabolites responsible for odour and colour of plants (3).

The concentrations of essence oil within plants vary according to stage of growth, stress, plant health and environmental factors such as light, temperature and moisture etc. (4). Essence oils are found throughout the plants, including roots, the bark, flowers, petals, leaves, fruit bodies and stems. Essence oils are the volatile components responsible for the characteristic aroma of spices. As active components in many spices and preservatives, essence oils have historically been used to inhibit bacterial growth (5). All essence oils have aromatic properties. They have also shown biological activities, such as antioxidative (6), antifungal (7) and antimicrobial activities (8), Furthermore they have been found to have psychological and physical effects, which is why they have found their application in aromatherapy.

One of the alternative to antibiotic feed supplements are plant extracts. In this study, Oreganum vulgare, Thymus vulgaris, Zingiber officinale, Syszygium aromaticum essence oils were supplemented to TMR, concentrate and hay to explore their effects on the levels of volatile fatty acid (VFA) concentrations at 8 and $24 \mathrm{~h}$ in in vitro conditions.

\section{INTRODUCCIÓN}

Las pérdidas de proteína y energía en la fermentación ruménica reducen el eficaz aprovechamiento de nutrientes y causan contaminación ambiental. Los antibióticos ionóforos se han utilizado en el rumen por muchos años para reducir estas pérdidas (1). Mientras que los antibióticos ionóferos se han utilizado ampliamente en los sistemas de producción convencional para el control de la fermentación ruménica, la Comisión Europea (CE) decidió eliminar gradualmente y en última instancia prohibir el uso de estos antibióticos (Enero $1^{\circ}$ 2006) (2). Después de la prohibición de los antibióticos promotores de crecimiento, han aumentado los estudios para buscar alternativas a éstos. Una de estas alternativas son los extractos vegetales, llamados también aceites esenciales. Estos aceites son metabolitos vegetales secundarios responsables del color y olor de las plantas (3).

La concentración de los aceites esenciales en las plantas varía de acuerdo con la etapa de crecimiento, estrés, salud de la planta y factores ambientales tales como la luz, temperatura, y humedad, etc. (4). Los aceites se encuentran en toda la planta, incluyendo raíces, corteza, flores, pétalos, hojas, frutos y tallos; y son los componentes volátiles responsables del aroma característico de las especias. Históricamente estos aceites se han utilizado para inhibir el crecimiento bacterial, como componentes activos de muchas especias y conservantes (5). Todos los aceites esenciales tienen propiedades aromáticas. Presentan también actividades biológicas, como antioxidantes (6), antifúngicos (7) y antimicrobianos. Así mismo, se observó que tienen efectos físicos y sicológicos, por lo cual han encontrado aplicación en aromaterapia.

Una de las alternativas a los suplementos del forraje basados en antibióticos, son los extractos vegetales. En este estudio, se agregaron aceites esenciales de Oreganum vulgare, Thymus vulgaris, Zingiber officinale, Syszygium aromaticum a la RMT, concentrado y heno para explorar sus efectos sobre las concentraciones de los niveles de ácidos grasos volátiles (AGV) a las 8 y 24 horas en condiciones in vitro. 


\section{MATERIAL AND METHODS}

Animals and feeds. Three fistulated Holstain dairy cows were used for rumen fluid collection for application of in vitro gas production technique. For each feed (TMR, Concentrate and Hay), ruminal fluid samples were collected twice daily on different days. Four essence oils ( $T$. vulgaris, $O$. vulgare, S. aromaticum, $Z$. officinale) were used as plant extracts. $T$. vulgaris, $S$. aromaticum, and $Z$. officinale essence oils were obtained from Manisa Province (Turkey) and O. vulgare essence oil from Mersin Province (Turkey). All plant extracts were extracted with distilated water. For each extract, different doses were tested to determine harmful and usable doses. Incubation was run for each regulation in 8 and $24 \mathrm{~h}$ time periods. Total mixed ration (TMR), concentrate and hay were used as substrates. In the first phase of the experiment, gasification quantity was measured. This step was iterated for the effects of each plant extract and each dose on substrates (TMR, concentrate, hay). After gasification measurements, harmful extract doses and critical time periods were identified. In the second phase of the experiment, VFA levels were measured.

The group with only rumen liquor was assigned as the negative control group and the group with rumen fluid and feed samples was assigned as the positive control group. The groups with rumen fluid, feed samples and thymol, oregano, zingiber and syzygium essence oils were the treatment groups. The compositions of TMR, concentrate and hay used in the experiment are presented respectively in table 1 . The effects of Syzygim aromaticum, Zingiber officinale, Thymus vulgaris, Oreganum vulagare supplementation to TMR, concentrate and hay on VFA production were assessed in the present experiment by incubation of in buffered rumen fluid using an in vitro gas production technique (HFT).

Sample preparation for VFA. Subsequent to the preparation of samples on experiment day, 1:11 with an internal Standard (4-Methylvalerianic

Table 1. Composition of TMR, Concentrate and Alfalfa

\begin{tabular}{lccc}
\multicolumn{2}{c}{ hay. } & & \\
\hline & Dry matter & Crude protein & MJNEL/ kg DM \\
\hline TMR $^{*}$ & 43.00 & 15.25 & 6.9 \\
Concentrate $* *$ & 98.50 & 20.30 & 8.7 \\
Alfalfa Hay & 95.31 & 14.46 & 8.0 \\
\hline
\end{tabular}

*TMR composition: Maize silage $28.9 \%$, Grass silage $31.2 \%$, Hay $4.8 \%$, Haylage $2.3 \%$, Molassed sugar beet pulp $1.3 \%$, Josera-betavit $0.09 \%$, Salt $0.09 \%$, Monosodium phosphate $0.09 \%$, Ca-carbonate $0.35 \%$, Bergophor GM13 0.45\%, Bergophor LM07 0.45\%, MgO 0.02\%, Concentrate-TMR-silage- $2,17.4 \%$, Soybean meals $0.7 \%$, chelates $0.3 \%$, Water $12.1 \%$, Yeast mixture $100 \mathrm{~g}$. Mineral g/kg DM: Ca 7.62, P 4.53, Mg 2.52, $\mathrm{Na} 2.82, \mathrm{~K} 16.04, \mathrm{Zn} \mathrm{62,} \mathrm{Mn} 58$ and Se 0.1.

$* *$ Concentrate composition: Maize $20 \%$, sunflower $7.5 \%$, pea $8 \%$, barley $16 \%$, soyabean meal $15 \%$, wheat $30.5 \%$, crib bean $3 \%$.

\section{MATERIALES Y MÉTODOS}

Animales y forrajes. Para la recolección del fluido ruménico y la aplicación de la técnica de producción de gas in vitro, se utilizaron tres vacas lecheras Holstein fistuladas. Para cada forraje (RMT, concentrado y heno) se colectaron las muestras de fluido ruminal dos veces por día en diferentes días. Se utilizaron como extractos vegetales cuatro aceites esenciales ( $T$. vulgaris, $O$. vulgare, $S$. aromaticum, $Z$. officinale). Los aceites esenciales de $T$. vulgaris, $S$. aromaticum, y $Z$. officinale se obtuvieron de la Provincia Manisa (Turquía) y el aceite esencial de $O$. vulgare de la Provincia Mersin (Turquía). Todos los extractos vegetales se extrajeron con agua destilada. Para cada extracto, se ensayaron diferentes dosis para determinar las útiles y las perjudiciales. La incubación se ejecutó para cada regulación en períodos de tiempo de 8 y 24 horas. Se utilizaron como sustratos la ración mixta total (RMT), concentrado y heno. En la primera fase del experimento, se midió la cantidad gasificada. Esta etapa se repitió para conocer los efectos de cada extracto vegetal y cada dosis sobre los sustratos (RMT, concentrado, heno). Después de medir la gasificación, se identificaron las dosis perjudiciales del extracto y los períodos críticos de tiempo. En la segunda fase del experimento se midieron los niveles de AGV.

Se asignó el grupo con líquido ruminal al grupo de control negativo y el grupo con fluido ruminal y muestras de forraje al grupo de control positivo. Los grupos de tratamiento fueron los que contenían fluido ruminal, muestras de forraje y timol, orégano, aceites esenciales de zingiber y syzygium. Las composiciones de RMT, concentrado y heno utilizadas en el experimento se presentaron respectivamente en la tabla 1 . En este experimento se evaluaron los efectos de la suplementación de Syzygim aromaticum, Zingiber officinale, Thymus vulgaris, Oreganum vulagarea a la RMT, concentrado y heno sobre la producción de AGV por incubación de fluido ruminal buffer (tampón), utilizando la técnica de producción de gas in vitro (HFT).

Preparación de muestras para AGV. Con el fin de medir los ácidos acético, propiónico, i-butírico y n-butírico, así como los ácidos i-valeriánico y n-valeriánico, en un día de experimento, posterior a la preparación de muestras, se preparó en una hora, 1:11 con un Estándar interno (ácido 4-Metilvaleriánico y ácido fórmico 1:101). Las muestras se congelaron a $-20^{\circ} \mathrm{C}$. Antes de la determinación de los ácidos grasos, estas muestras se descongelaron a temperatura ambiente y se centrifugaron a $400 \mathrm{~g}$ por 10 minutos y después se tomaron las partes claras para realizar las mediciones. 
acid and formic acid 1:101) was prepared in an hour for measurement of acetic, propionic, i-butiric and $n$-butiric acid as well as i-valerianic and $n$-valerianic acid. The samples were frozen at $-20^{\circ} \mathrm{C}$. Before determination of the fatty acids, the samples were thawed at room temperature and centrifuged in $400 \mathrm{~g}$ for ten minutes and afterwards, bright parts were taken for measurements.

Analysis. Concentrations of VFAs were analysed in pooled samples of each replicate, using a gas chromatograph (GC 14B, Shimadzu, Japan) equipped with a flame ionisation detector, and samples were treated with an Internal Standard (4-Methylvalerianic acid and formic acid 1:101) according to Geissler et al (9). A total of 6 parallel analyses were performed for each sample.

Statistical analysis. The descriptive statistics for the examined parameters were expressed in terms of average and standard errors. Factorial Analysis of Variance (Factorial ANOVA) was conducted to determine any differences between the means of supplements and feed types with respect to the examined parameters. Additionally, Repeated Measures ANOVA was performed to determine any differences with respect to feeds and durations (hours). Following analyses of variance, Tukey test was conducted to determine varying means (10). Statistical significance level was set at $5 \%$ and calculations were performed by SPSS (11) statistical software package.

\section{RESULTS}

The descriptive statistics of some VFAs at 8 and $24 \mathrm{~h}$ according to supplement and feed groups and their comparative results according to supplement groups are given in tables 2 and 3 .

As seen in table 2, compared to negative control groups, essence oil supplementations had significant effect on C2, C3, IC4, C4 IC5, $\mathrm{C} 5$ and C2/C3 VFA level at $8 \mathrm{~h}(\mathrm{p}<0.05)$. For TMR, Concentrate and hay group, C2, C3, and C4 VFA levels increased at $8 \mathrm{~h}$ in the treatment groups with essence oil supplementations compared to negative control group $(p<0.05)$. On the contrary, for TMR, C4 VFA level decreased in the group with Syzygium 200 supplementation with respect to positive control group $(p<0.05)$. For concentrate group, C4 VFA level decreased at $8 \mathrm{~h}$ in the group with Syzygium 200 supplementation compared to positive control groups $(p<0.05)$. For hay group, C4 VFA level increased at $8 \mathrm{~h}$ in the other treatment groups with respect to negative control group $(p<0.05)$.
Análisis. Se analizaron las concentraciones de AGV en muestras combinadas de cada réplica, utilizando cromatógrafo de gases (GC 14B, Shimadzu, Japón) equipado con un detector de ionización de llama y las muestras se trataron con un Estándar interno (ácido 4-Metilvaleriánico y ácido fórmico 1:101) de acuerdo con Geissler et al (9). Se llevaron a cabo un total de 6 análisis paralelos para cada muestra.

Análisis Estadístico. Las estadísticas descriptivas para los parámetros examinados se expresaron en términos de errores estándar y promedio. Se realizó el Análisis Factorial de Varianza (ANOVA Factorial) para determinar algunas diferencias entre el promedio de suplementos y los tipos de forraje, con respecto a los parámetros examinados. Adicionalmente, se llevó a cabo el ANOVA de Medidas Repetidas para determinar algunas diferencias con respecto a los forrajes y duraciones (horas). Tras el análisis de varianza, se efectuó el ensayo Tukey para determinar los medios de varianza (10), Se estableció un nivel estadísticamente significante en $5 \%$ y los cálculos se realizaron mediante el paquete de programas estadísticos SPSS (11).

\section{RESULTADOS}

En las tablas 2 y 3 se presentan las estadísticas descriptivas de algunos AGV a 8 y 24 horas, de acuerdo con el grupo de forraje y suplemento y sus resultados comparativos según los grupos de suplemento.

Como se ve en la tabla 2, las suplementaciones con aceite esencial tuvieron un efecto significativo sobre los niveles AGV C2, C3, IC4, C4 IC5, C5 y C2/C3 a las 8 horas $(p<0.05)$ comparado con los grupos de control negativo. Para el grupo RMT, concentrado y ceno, se incrementaron los niveles AGV C2, C3 y C4 a las 8 horas en los grupos de tratamiento con suplementaciones de aceite esencial comparado con el grupo de control negativo $(p<0.05)$. Por el contrario, para RMT, el nivel AGV C4 disminuyó en el grupo con suplementación de syzygium 200 con respecto al grupo de control positivo $(\mathrm{p}<0.05)$. Para el grupo concentrado, el nivel AGV C4 disminuyó a las 8 horas en el grupo con suplementación de syzygium 200 comparado con los grupos de control positivo $(p<0.05)$. Para el grupo heno, el nivel AGV C4 aumentó a las 8 horas en los otros grupos de tratamiento con respecto al grupo de control negativo $(p<0.05)$.

La tabla 3 indica que las suplementaciones con aceite esencial tuvieron un efecto significativo sobre los niveles AGV C2, C3, IC4, IC5, C5 y C2/ C3 a las 24 horas $(p<0.05)$. La concentración de ácido graso volátil C3 aumentó significativamente en los grupos RMT y heno con suplementaciones 
Table 2. Descriptive statistics of some VFAs at $8 \mathrm{~h}$ according to supplement and feed groups and their comparative results according to supplement groups.

\begin{tabular}{|c|c|c|c|c|c|c|c|c|}
\hline \multirow[b]{2}{*}{ Feed } & \multirow[b]{2}{*}{ Supplements } & \multicolumn{7}{|c|}{ Volatile Fatty Acids, $\mathrm{mmol} / \mathrm{I}$} \\
\hline & & $\begin{array}{c}\mathrm{C2} \\
\text { (Acetic A) }\end{array}$ & $\begin{array}{c}\text { C3 } \\
\text { (Propionic A) }\end{array}$ & $\begin{array}{c}\text { IC4 } \\
\text { (Isobutyric A) }\end{array}$ & $\begin{array}{c}\text { C4 } \\
\text { (Butyric A) }\end{array}$ & $\begin{array}{c}\text { IC5 } \\
\text { (Isovaleric A) }\end{array}$ & $\begin{array}{c}\text { C5 } \\
\text { (Valeric A) }\end{array}$ & $\mathrm{C} 2 / \mathrm{C} 3$ \\
\hline \multirow{8}{*}{ TMR } & Negative control & $39.30 \pm 0.47 c$ & $8.77 \pm 0.05 c$ & $0.91 \pm 0.01 \mathrm{abc}$ & $9.56 \pm 0.15 d$ & $1.47 \pm 0.02 a$ & $0.82 \pm 0.01 b$ & $4.48 \pm 0.02 c$ \\
\hline & Positive control & $51.47 \pm 0.45 a$ & $12.96 \pm 0.11 \mathrm{a}$ & $0.95 \pm 0.01 \mathrm{abc}$ & $12.59 \pm 0.01 \mathrm{ab}$ & $1.44 \pm 0.00 a$ & $1.05 \pm 0.00 \mathrm{ab}$ & $3.97 \pm 0.00 \mathrm{~d}$ \\
\hline & Thymol 12.5 & $50.63 \pm 1.07 a$ & $12.64 \pm 0.22 a b$ & $1.04 \pm 0.03 a$ & $12.77 \pm 0.15 \mathrm{ab}$ & $1.84 \pm 0.07 a$ & $1.13 \pm 0.03 a$ & $4.01 \pm 0.01 d$ \\
\hline & Thymol 25 & $45.14 \pm 2.33 b$ & $9.51 \pm 0.42 c$ & $0.75 \pm 0.04 c$ & $11.71 \pm 0.50 b c$ & $1.48 \pm 0.11 \mathrm{a}$ & $0.94 \pm 0.04 a b$ & $4.75 \pm 0.02 b$ \\
\hline & Oregano 12.5 & $50.66 \pm 1.24 a$ & $12.50 \pm 0.03 a b$ & $1.01 \pm 0.03 a b$ & $12.86 \pm 0.21 \mathrm{ab}$ & $1.72 \pm 0.17 a$ & $1.10 \pm 0.04 a$ & $4.05 \pm 0.08 d$ \\
\hline & Oregano 25 & $50.81 \pm 0.70 a$ & $10.35 \pm 0.15 b c$ & $0.82 \pm 0.01 b c$ & $12.78 \pm 0.03 a b$ & $1.70 \pm 0.01 a$ & $1.02 \pm 0.01 \mathrm{ab}$ & $4.91 \pm 0.00 a$ \\
\hline & Zingiber 200 & $53.60 \pm 0.45 a$ & $13.74 \pm 0.01 \mathrm{a}$ & $0.98 \pm 0.01 \mathrm{ab}$ & $13.42 \pm 0.06 a$ & $1.50 \pm 0.01 a$ & $1.13 \pm 0.00 a$ & $3.90 \pm 0.03 d$ \\
\hline & Syzygium 200 & $45.19 \pm 0.40 b$ & $14.70 \pm 0.48 a$ & $0.76 \pm 0.02 c$ & $10.84 \pm 0.30 c$ & $1.49 \pm 0.06 a$ & $1.05 \pm 0.09 \mathrm{ab}$ & $3.08 \pm 0.10 \mathrm{e}$ \\
\hline \multirow{8}{*}{ Concentrate } & Negative control & $39.30 \pm 0.47 d$ & $8.77 \pm 0.05 b$ & $0.91 \pm 0.01 \mathrm{ab}$ & $9.56 \pm 0.15 d$ & $1.47 \pm 0.02 \mathrm{abc}$ & $0.82 \pm 0.01 \mathrm{c}$ & $4.48 \pm 0.02 b c$ \\
\hline & Positive control & $55.74 \pm 2.33 a b$ & $12.83 \pm 0.55 a$ & $0.87 \pm 0.02 a b c$ & $13.39 \pm 0.51 \mathrm{ab}$ & $1.16 \pm 0.01 \mathrm{c}$ & $1.09 \pm 0.04 a b c$ & $4.35 \pm 0.00 b c$ \\
\hline & Thymol 12.5 & $56.59 \pm 0.81 a b$ & $13.32 \pm 0.01 a$ & $0.88 \pm 0.01 a b c$ & $13.67 \pm 0.28 a b$ & $1.33 \pm 0.01 b c$ & $1.11 \pm 0.01 \mathrm{ab}$ & $4.24 \pm 0.02 b c$ \\
\hline & Thymol 25 & $56.83 \pm 6.05 a b$ & $12.75 \pm 4.42 a$ & $0.94 \pm 0.30 a$ & $13.35 \pm 0.93 a b$ & $1.84 \pm 0.60 a$ & $1.26 \pm 0.46 a$ & $4.66 \pm 0.81 b$ \\
\hline & Oregano 12.5 & $58.12 \pm 1.21 a$ & $14.61 \pm 1.57 a$ & $1.02 \pm 0.15 a$ & $13.86 \pm 0.28 a$ & $1.78 \pm 0.57 a b$ & $1.31 \pm 0.23 a$ & $4.00 \pm 0.25 b c$ \\
\hline & Oregano 25 & $52.48 \pm 3.61 b c$ & $9.65 \pm 0.64 b$ & $0.72 \pm 0.04 b c$ & $12.62 \pm 1.70 b$ & $1.39 \pm 0.11 \mathrm{abc}$ & $0.91 \pm 0.07 b c$ & $5.44 \pm 0.01 a$ \\
\hline & Zingiber 200 & $58.88 \pm 0.43 a$ & $13.86 \pm 0.10 a$ & $0.91 \pm 0.01 \mathrm{ab}$ & $14.46 \pm 0.06 a$ & $1.21 \pm 0.00 c$ & $1.19 \pm 0.01 \mathrm{a}$ & $4.25 \pm 0.00 b c$ \\
\hline & Syzygium 200 & $48.95 \pm 3.26 c$ & $13.52 \pm 0.95 a$ & $0.69 \pm 0.04 c$ & $10.10 \pm 0.77 c$ & $1.33 \pm 0.08 \mathrm{bc}$ & $0.88 \pm 0.06 \mathrm{bc}$ & $3.62 \pm 0.01 \mathrm{c}$ \\
\hline \multirow{8}{*}{ Hay } & Negative control & $36.85 \pm 0.26 c$ & $8.84 \pm 0.39 c$ & $0.88 \pm 0.01 a b$ & $8.62 \pm 0.05 b$ & $1.39 \pm 0.01 \mathrm{abc}$ & $0.79 \pm 0.01 a$ & $4.17 \pm 0.11 b c$ \\
\hline & Positive control & $45.60 \pm 0.60 a b$ & $11.16 \pm 0.20 b c$ & $0.85 \pm 0.01 \mathrm{ab}$ & $10.02 \pm 0.11 a$ & $1.26 \pm 0.00 c$ & $0.89 \pm 0.01 a$ & $4.09 \pm 0.01 b c$ \\
\hline & Thymol 12.5 & $47.26 \pm 0.00 \mathrm{a}$ & $11.78 \pm 0.00 \mathrm{ab}$ & $0.99 \pm 0.00 a$ & $10.60 \pm 0.00 a$ & $1.74 \pm 0.00 \mathrm{ab}$ & $0.97 \pm 0.00 a$ & $4.01 \pm 0.00 c$ \\
\hline & Thymol 25 & $43.15 \pm 2.05 a b$ & $9.36 \pm 0.41 b c$ & $0.78 \pm 0.04 b$ & $9.93 \pm 0.14 a$ & $1.44 \pm 0.14 \mathrm{abc}$ & $0.89 \pm 0.04 a$ & $4.61 \pm 0.01 a$ \\
\hline & Oregano 12.5 & $46.12 \pm 0.00 \mathrm{ab}$ & $11.56 \pm 0.00 \mathrm{~b}$ & $1.00 \pm 0.00 a$ & $10.40 \pm 0.09 a$ & $1.81 \pm 0.02 a$ & $0.99 \pm 0.01 a$ & $3.99 \pm 0.00 c$ \\
\hline & Oregano 25 & $42.93 \pm 5.39 a b$ & $9.91 \pm 1.92 b c$ & $0.89 \pm 0.25 a b$ & $9.84 \pm 0.83 a$ & $1.65 \pm 0.58 \mathrm{ab}$ & $0.98 \pm 0.28 a$ & $4.36 \pm 0.21 b$ \\
\hline & Zingiber 200 & $45.94 \pm 0.03 a b$ & $11.66 \pm 0.04 a b$ & $0.86 \pm 0.00 \mathrm{ab}$ & $10.39 \pm 0.01 a$ & $1.29 \pm 0.01 b c$ & $0.95 \pm 0.01 a$ & $3.94 \pm 0.01 \mathrm{c}$ \\
\hline & Syzygium 200 & $42.24 \pm 0.37 b$ & $14.01 \pm 0.12 \mathrm{a}$ & $0.78 \pm 0.01 b$ & $10.04 \pm 0.21 a$ & $1.52 \pm 0.05 \mathrm{abc}$ & $0.98 \pm 0.03 a$ & $3.02 \pm 0.00 \mathrm{~d}$ \\
\hline
\end{tabular}

Table 3. Descriptive statistics of some VFAs at $24 \mathrm{~h}$ according to supplement and feed groups and their comparative results according to supplement groups.

\begin{tabular}{|c|c|c|c|c|c|c|c|c|}
\hline \multirow[b]{2}{*}{ Feed } & \multirow[b]{2}{*}{ Supplements } & \multicolumn{7}{|c|}{ Volatile Fatty Acids, mmol/l $\quad \bar{X} \pm S_{\bar{x}}$} \\
\hline & & $\begin{array}{c}\text { C2 } \\
\text { (Acetic A) }\end{array}$ & $\begin{array}{c}\text { C3 } \\
\text { (Propionic A) }\end{array}$ & $\begin{array}{c}\text { IC4 } \\
\text { (Isobutyric A) }\end{array}$ & $\begin{array}{c}\text { C4 } \\
\text { (Butyric A) }\end{array}$ & $\begin{array}{c}\text { IC5 } \\
\text { (Isovaleric A) }\end{array}$ & $\begin{array}{c}\text { C5 } \\
\text { (Valeric A) }\end{array}$ & $\mathrm{C2} / \mathrm{C} 3$ \\
\hline \multirow{8}{*}{ TMR } & Negative control & $48.61 \pm 0.42 e$ & $11.23 \pm 0.08 \mathrm{~d}$ & $1.55 \pm 0.00 a$ & $12.89 \pm 0.11 \mathrm{c}$ & $3.07 \pm 0.01 a$ & $1.42 \pm 0.01 \mathrm{de}$ & $4.33 \pm 0.00 c$ \\
\hline & Positive control & $71.15 \pm 1.23 a$ & $17.03 \pm 0.36 b c$ & $1.69 \pm 0.01 a$ & $18.27 \pm 0.04 a b$ & $3.07 \pm 0.03 a$ & $1.81 \pm 0.01 \mathrm{ab}$ & $4.18 \pm 0.01 d$ \\
\hline & Thymol 12.5 & $71.32 \pm 2.60 a$ & $17.27 \pm 0.76 b$ & $1.72 \pm 0.05 a$ & $18.53 \pm 0.64 a b$ & $3.13 \pm 0.09 a$ & $1.84 \pm 0.07 a b$ & $4.13 \pm 0.02 d$ \\
\hline & Thymol 25 & $57.09 \pm 5.55 \mathrm{~cd}$ & $9.93 \pm 0.91 \mathrm{e}$ & $0.81 \pm 0.09 c$ & $19.13 \pm 0.18 \mathrm{a}$ & $1.86 \pm 030 b$ & $1.19 \pm 0.04 f$ & $5.75 \pm 0.02 a$ \\
\hline & Oregano 12.5 & $67.24 \pm 0.31 \mathrm{ab}$ & $16.03 \pm 0.11 \mathrm{c}$ & $1.59 \pm 0.01 \mathrm{a}$ & $18.21 \pm 0.04 a b$ & $3.15 \pm 0.02 a$ & $1.66 \pm 0.02 b c$ & $4.20 \pm 0.03 d$ \\
\hline & Oregano 25 & $62.16 \pm 1.06 \mathrm{bc}$ & $11.28 \pm 0.27 d$ & $1.27 \pm 0.06 \mathrm{~b}$ & $17.91 \pm 0.32 \mathrm{ab}$ & $3.00 \pm 0.08 a$ & $1.34 \pm 0.05 \mathrm{ef}$ & $5.51 \pm 0.03 b$ \\
\hline & Zingiber 200 & $70.06 \pm 0.90 a$ & $19.43 \pm 0.10 a$ & $1.65 \pm 0.03 a$ & $19.06 \pm 0.11 a$ & $3.02 \pm 0.03 a$ & $1.95 \pm 0.01 a$ & $3.61 \pm 0.05 \mathrm{e}$ \\
\hline & Syzygium 200 & $52.85 \pm 0.45 \mathrm{de}$ & $20.33 \pm 0.82 a$ & $0.81 \pm 0.05 c$ & $16.03 \pm 0.08 b$ & $1.78 \pm 0.13 b$ & $1.60 \pm 0.07 \mathrm{~cd}$ & $2.61 \pm 0.06 f$ \\
\hline \multirow{8}{*}{ Concentrate } & Negative control & $45.08 \pm 0.16 c$ & $10.54 \pm 0.04 d$ & $1.42 \pm 0.00 a$ & $11.78 \pm 0.04 c$ & $2.66 \pm 0.00 a$ & $1.26 \pm 0.00 \mathrm{~b}$ & $4.28 \pm 0.00 b$ \\
\hline & Positive control & $69.99 \pm 0.28 a$ & $16.18 \pm 0.04 b$ & $1.54 \pm 0.01 a$ & $17.89 \pm 0.08 \mathrm{ab}$ & $2.53 \pm 0.01 a$ & $1.77 \pm 0.00 a$ & $4.33 \pm 0.00 b$ \\
\hline & Thymol 12.5 & $67.12 \pm 1.30 a$ & $16.21 \pm 0.12 b$ & $1.43 \pm 0.08 a$ & $17.18 \pm 0.28 a b$ & $2.59 \pm 0.05 a$ & $1.67 \pm 0.11 a$ & $4.14 \pm 0.08 b$ \\
\hline & Thymol 25 & $57.83 \pm 2.54 b$ & $9.89 \pm 0.54 d$ & $0.90 \pm 0.06 b$ & $18.64 \pm 0.20 a$ & $1.96 \pm 0.21 b c$ & $1.33 \pm 0.05 b$ & $5.85 \pm 0.04 a$ \\
\hline & Oregano 12.5 & $67.92 \pm 1.19 a$ & $16.18 \pm 0.19 b$ & $1.46 \pm 0.06 a$ & $17.29 \pm 0.62 a b$ & $2.59 \pm 0.03 a$ & $1.69 \pm 0.10 a$ & $4.20 \pm 0.09 b$ \\
\hline & Oregano 25 & $57.64 \pm 8.67 b$ & $10.21 \pm 1.90 d$ & $1.08 \pm 0.41 b$ & $15.39 \pm 4.33 b$ & $2.19 \pm 1.05 a b$ & $1.21 \pm 0.40 \mathrm{~b}$ & $5.67 \pm 0.15 a$ \\
\hline & Zingiber 200 & $67.85 \pm 1.16 a$ & $18.47 \pm 0.14 a$ & $1.48 \pm 0.04 a$ & $17.99 \pm 0.44 a$ & $2.50 \pm 0.06 a$ & $1.85 \pm 0.05 a$ & $3.67 \pm 0.02 c$ \\
\hline & Syzygium 200 & $50.26 \pm 0.21 \mathrm{c}$ & $14.94 \pm 0.06 c$ & $0.77 \pm 0.03 c$ & $11.18 \pm 0.04 \mathrm{c}$ & $1.55 \pm 0.03 c$ & $0.96 \pm 0.00 c$ & $3.37 \pm 0.00 d$ \\
\hline \multirow{8}{*}{ Hay } & Negative control & $44.96 \pm 2.37 c$ & $10.87 \pm 0.17 d$ & $1.51 \pm 0.05 a$ & $11.39 \pm 1.24 \mathrm{bcd}$ & $2.96 \pm 0.15 a$ & $1.33 \pm 0.04 b$ & $4.14 \pm 0.09 c$ \\
\hline & Positive control & $64.14 \pm 2.46 a$ & $16.85 \pm 0.28 b$ & $1.57 \pm 0.01 a$ & $14.82 \pm 1.61 \mathrm{a}$ & $2.90 \pm 0.04 a$ & $1.61 \pm 0.02 \mathrm{a}$ & $3.81 \pm 0.10 c$ \\
\hline & Thymol 12.5 & $58.89 \pm 1.80 a b$ & $14.92 \pm 0.45 c$ & $1.40 \pm 0.07 a b$ & $13.42 \pm 0.86 \mathrm{ab}$ & $2.67 \pm 0.13 a$ & $1.37 \pm 0.10 b$ & $3.95 \pm 0.08 c$ \\
\hline & Thymol 25 & $45.46 \pm 6.84 c$ & $9.02 \pm 0.84 \mathrm{e}$ & $0.75 \pm 0.10 c$ & $10.62 \pm 1.75 \mathrm{~cd}$ & $1.54 \pm 0.35 b$ & $0.91 \pm 0.13 d$ & $5.02 \pm 0.17 a$ \\
\hline & Oregano 12.5 & $55.01 \pm 1.16 b$ & $14.28 \pm 0.14 c$ & $1.31 \pm 0.00 \mathrm{~b}$ & $12.12 \pm 0.23 \mathrm{bcd}$ & $2.52 \pm 0.00 a$ & $1.27 \pm 0.03 b c$ & $3.85 \pm 0.03 c$ \\
\hline & Oregano 25 & $44.30 \pm 0.41 c$ & $9.54 \pm 0.08 \mathrm{e}$ & $0.76 \pm 0.05 c$ & $9.96 \pm 0.03 b$ & $1.40 \pm 0.00 \mathrm{~b}$ & $0.95 \pm 0.01 d$ & $4.65 \pm 0.00 b$ \\
\hline & Zingiber 200 & $56.97 \pm 0.35 b$ & $18.29 \pm 0.42 a$ & $1.48 \pm 0.01 \mathrm{ab}$ & $12.73 \pm 0.07 a b c$ & $2.69 \pm 0.01 a$ & $1.59 \pm 0.01 a$ & $3.12 \pm 0.04 d$ \\
\hline & Syzygium 200 & $43.32 \pm 0.33 c$ & $18.80 \pm 0.43 a$ & $0.74 \pm 0.02 c$ & $9.65 \pm 0.06 b$ & $1.54 \pm 0.01 b$ & $1.11 \pm 0.02 \mathrm{~cd}$ & $2.31 \pm 0.03 e$ \\
\hline
\end{tabular}

*: Supplement averages (within each feed group) with different letters denote statistically significant differences $(p<0.05)$. 
Table 3 indicates that essence oil supplementations had significant effect on C2, C3, IC4, IC5, C5 and C2/C3 VFA levels at $24 \mathrm{~h}(\mathrm{p}<0.05)$. C3 volatile fatty acid concentration significantly increased in TMR and hay groups with 200 ppm of Zingiber and Syzygium supplementations $(p<0.05) .200$ ppm of Syzygium supplementation significantly decreased propionic acid concentration for concentrate $(p<0.05)$. In all feed groups, IC4 VFA levels significantly decreased with Thymol 25, Oregano 25 and Syzygium 200 essence oil supplementations compared to positive and negative control groups $(p<0.05)$. IC5 VFA levels also significantly decreased in all feed groups with Thymol 25 and Syzygium 200 essence oil supplementations compared to positive and negative control groups $(p<0.05)$. C5 VFA level significantly decreased in TMR group supplemented with Thymol 25 , in concentrate group supplemented with Syzygium 200 and in hay groups supplemented with Thymol 25, Oregano 25 and Syzygium 200 essence oils $(p<0.05)$. C2/C3 VFA level at $24 \mathrm{~h}$ significantly increased with $25 \mathrm{ppm}$ dose of Thymol and Oregano extracts and significantly decreased with 200 ppm dose of Zingiber and Syzygium for all feed groups (TMR, concentrate and hay).

\section{DISCUSSION}

Effects of essence oils on VFA. As a result of the microbial activity in the rumen of ruminants, carbohydrates such as cellulose are broken down and converted to VFAs, carbon dioxide, methane, ammonia and microbial cells (12). VFAs are the main energy source of ruminants. About $70 \%$ of the metabolizable energy in ruminants is generated by the VFAs produced in the rumen (13). If reduction of VFA concentrations in rumen at in vitro conditions occurs also at in vivo conditions, this situation may adversely affect nutrition efficiency (14). In this study, the effects of thymol, oregano, zingiber and syzygium supplementations to TMR, concentrate and hay at 8 and $24 \mathrm{~h}$ were examined in in vitro conditions.

As seen in table 2, compared to positive and negative control groups, essence oil supplementations did not have any statistically significant effect at $8 \mathrm{~h}$ on isobutyric acid, isovaleric acid and valaric acid concentrations in the examined feed groups. Beauchemin and McGinn (15) reported no effects of 750 $\mathrm{mg} / \mathrm{d}$ essence oil on total VFA production $4 \mathrm{~h}$ post feeding in cattle. Benchaar et al. (16) demonstrated no effects of thymol, eugenol, de 200 ppm de zingiber y syzygium $(p<0.05)$. Para el concentrado, la suplementación de 200 ppm de syzygium disminuyó significativamente la concentración de ácido propiónico $(p<0.05)$. En todos los grupos de forraje, los niveles AGV IC4 disminuyeron significativamente con suplementaciones de aceite esencial de timol 25, orégano 25 y syzygium 200, comparado con los grupos de control positivo y negativo $(p<0.05)$. En todos los grupos de forraje el nivel AGV IC5 también disminuyó significativamente con suplementaciones de aceites esenciales timol 25 y syzygium 200 comparado con los grupos de control positivo y negativo $(p<0.05)$. En el grupo RMT suplementado con timol 25, el grupo concentrado suplementado con syzygium 200 y el grupo heno suplementado con los aceites esenciales de timol 25, orégano 25 y syzygium 200, el nivel AGV IC5 disminuyó significativamente $(p<0.05)$. En todos los grupos de forraje (RMT, concentrado y heno), el nivel AGV C2/ C3 a las 24 horas aumentó significativamente con dosis de 25 ppm de timol y extractos de orégano y disminuyó significativamente con dosis de 200 ppm de zingiber y syzygium.

\section{DISCUSIÓN}

Efectos de los aceites esenciales sobre los AGV. Como resultado de la actividad microbiana en el rumen de los rumiantes, los carbohidratos tales como celulosa se rompen y se convierten en AGV, dióxido de carbono, metano, amoniaco y células microbianas (12). Los AGV son la principal fuente de energía de los rumiantes. Cerca del $70 \%$ de la energía metabolizada por éstos se genera por la producción de AGV en el rumen (13). Si la reducción de las concentraciones de AGV en el rumen en condiciones in vitro ocurre también en condiciones in vivo, esta situación puede afectar negativamente la eficiencia de la nutrición (14). En este estudio, se analizaron los efectos de las suplementaciones con timol, orégano, zingiber y syzygium a la RMT, concentrado y heno a las 8 y 24 horas en condiciones in vitro.

Como se observa en la tabla 2 , en los grupos de forraje examinados, las suplementaciones con aceite esencial, en comparación con los grupos de control positivo y negativo, no tuvieron ningún efecto significativo a las 8 horas, sobre las concentraciones de los ácidos isobutírico e isovalérico. Beauchemin y McGinn (15) reportaron que $750 \mathrm{mg} / \mathrm{d}$ de aceite esencial no tiene efectos sobre la producción de AGV total, a las 4 horas posteriores a la alimentación del ganado. Benchaar et al (16) demostraron que no hay efectos en las vacas, de la combinación de aceites esenciales de timol, eugenol, vainilla y limoneno ( $2 \mathrm{~g} /$ día) sobre las concentraciones de AGV total e individual, a 
vanilin and limonen essence oil combination ( $2 \mathrm{~g} /$ day) on total VFA and individual VFA concentrations in cows at $8 \mathrm{~h}$. In the study of Chaves et al (17) on Holstein cows, carvacrol and cinnamaldeyhde supplementation at cattle ration did not have any effect on total VFA and individual VFA concentrations at 6 h. Kamalak et al (18) suggested that thymol has no effect on molar proportions of acetate, propionate, butyrate, isobutyrate, valerate and isovalerate. The findings of these researchers support the findings of this study which showed that essence oils have no significant effect on isobutyric, isovaleric and valeric acid VFAs at $8 \mathrm{~h}$.

As seen in table 2, essence oil supplementations had statistically significant effect at $8 \mathrm{~h}$ on butyric acid concentration $(p<0.05)$ according to feed. Compared to positive control groups, Syzygium 200 ppm supplementation resulted a significant decrease in butyric acid levels for TMR and concentrate $(p<0.05)$. Öztürk et al (19) revealed that $150 \mathrm{mg}$ of olive leaf extract reduced butyrate production. The findings of these researchers are in agreement with the findings obtained in this study. Contrary to the findings of this study, Castillejos et al (20) reported that $500 \mathrm{mg} / \mathrm{l}$ of thymol and eugenol supplementation to 60:40 roughage:concentrate ration significantly increased butyric acid concentration. Bencharr et al (14) demonstrated that carvacrol and eugenol increased butyrate proportions. García et al (21) collected rumen liquors in in vitro conditions from goats fed on 70:30 barley:alfalfa ration and found that $250 \mathrm{mg} / \mathrm{I}$ of carvacrol supplementation increased butyrate proportions after $48 \mathrm{~h}$ incubation. Chaves et al (22) observed that carvacrol or cinnamaldehyde supplementation to barley or corn grain-based lamb rations did not have any significant effect on butyrate proportions compared to negative control group. Sirohi et al (23) observed no effect of Myristica fragrans extract on butyrate proportions. As indicated by the literature, while some essence oils increase butyric acid concentrations, some essence oils decrease these concentrations and some essence oils have no effect on these concentrations. The varying results obtained from studies can be attributed to the ration compositions and types of plant extracts used in the studies as well as their extraction methods and doses.

Cardozo et al (24) reported that high doses (300 mg/l) of garlic, cinnamon, yucca, anise, oregano, capsicum essence oils and cinnamaldehyde, anethole, eugenol plant las 8 horas. En el estudio de Chaves et al (17) sobre las vacas Holstein, la suplementación con carvacrol y cinamaldehído en la ración del ganado, no tuvo ningún efecto sobre las concentraciones de AGV total e individual a las 6 horas. Kamalak et al (18) sugirieron que el timol no tiene efecto sobre las proporciones molares de acetato, propionato, butirato, isobutirato, valerato e isovalerato. Los resultados de estos investigadores apoyan los hallazgos de este estudio, los cuales mostraron que a las 8 horas, los aceites esenciales no tienen efecto significativo sobre los AGV, ácidos isobutírico, isovalérico y valérico.

En la tabla 2 se observa que de acuerdo con el forraje, las suplementación con aceite esencial tuvo efecto significativo a las 8 horas, sobre la concentración de ácido butírico $(p<0.05)$. La suplementación al RMT y concentrado con 200 ppm de syzygium produjo una disminución significativa en los niveles de ácido butírico $(p<0.05)$, en comparación con los grupos de control positivo. Öztürk et al (19) revelaron que 150 mg de extracto de hoja de olivo redujeron la producción de butirato. Los resultados de estos investigadores están de acuerdo con los obtenidos en este estudio. En contra de las conclusiones de este estudio, Castillejos et al (20) reportaron que la suplementación con 500 $\mathrm{mg} / \mathrm{l}$ de timol y eugenol en forraje: concentrado en una relación 60:40 aumentó significativamente la concentración de ácido butírico. Bencharr et al (14) demostraron que el carvacrol y el eugenol incrementaron las proporciones de butirato. Garcia et al (21) colectaron licor ruminal en condiciones in vitro de cabras alimentadas con cebada:alfalfa en proporción 70:30 y encontró que $250 \mathrm{mg} / \mathrm{l}$ de la suplementación con carvacrol incrementó las proporciones de butirato, después de 48 horas de incubación. Chaves et al (22) observaron que la suplementación con carvacrol y cinamaldehido de las raciones del cordero a base de cebada o maíz en grano, no tuvieron ningún efecto significativo sobre las proporciones de butirato en comparación con los grupos de control negativo. Sirohi et al (23) no observaron efecto del extracto de Myristica fragrans sobre las proporciones de butirato. Como se señala en la bibliografía, mientras algunos aceites esenciales aumentan las concentraciones de ácido butírico, algunos las disminuyen y algunos no tienen ningún efecto sobre estas concentraciones. Los resultados variables obtenidos de estos estudios, se pueden atribuir a las proporciones de las composiciones y a los tipos de extractos vegetales utilizados en dichos estudios, así como también a los métodos de extracción y dosis usados.

Cardozo et al (24) reportaron que altas dosis (300 mg/l) de aceites esenciales de ajo, canela, yuca, anís, orégano, pimiento y metabolitos secundarios vegetales de cinamaldehído, anetol, 
secondary metabolites used in their study significantly decreased total VFA concentrations at $24 \mathrm{~h}$. In the study of Busquet et al (25), $3.000 \mathrm{mg} / \mathrm{l}$ doses of carvacrol, clove bud, eugenol and oregano oil supplementation to 50:50 roughage:concentrate rations significantly decreased total VFA concentrations at $24 \mathrm{~h}$. Castillejos et al (20) demonstrated that $5.000 \mathrm{mg} / \mathrm{l}$ doses of thymol and eugenol supplementation to 60:40 roughage: concentrate rations significantly decreased total VFA concentrations at $24 \mathrm{~h}$. Petersen et al (26) observed that Larrea plant extract significantly decreased total VFA concentrations in $100 \%$ timothy and 50:50 alfalfa-corn rations at 48 h. Canpolat et al. (27) reported that varying doses of carvacrol, peppermint oil and orange oil supplementations to rumen liquors collected from rams fed on 60:40 alfalfa grassconcentrate ration significantly decreased total VFA, butyric acid and acetic acid/propionic acid concentrations. In our studies, for all feed groups, Thymol 25, Oregano 25, Syzygium 200 ppm supplementations significantly reduced isobuytric acid concentration at 24 h. Further, Thymol 25 and Syzygium 200 ppm supplementations significantly reduced isovaleric VFA concentration at 24 h compared to positive and negative control groups $(p<0.05)$. Castillejos et al $(20)$ observed that $500 \mathrm{mg} / \mathrm{l}$ of thymol and eugenol supplementations significantly decreased isovalerate and isobutytrate concentrations at $24 \mathrm{~h}$. The findings of these researchers support the findings of this study.

Similarly, at $24 \mathrm{~h}$, propionic acid concentration significantly decreased in the TMR, concentrate and hay groups with 25 ppm of Thymol supplementations compared to positive and negative control groups $(p<0.05)$. Isovaleric concentration significantly decreased particularly in Thymol 25 and Syzygium 200 ppm supplemented in TMR, concentrate and hay group compared to positive and negative control groups $(p<0.05)$. Acetic acid/propionic acid concentrations decreased significantly in Zingiber 200 ppm and Syzygium 200 supplemented TMR, concentrate and hay groups compared to positive and negative control groups. The study findings on VFA concentrations for feed groups at $24 \mathrm{~h}$ are in agreement with the findings of the researchers mentioned above. Decreased VFA concentrations in rumen liquor can be attributed to reduced activities of ruminal microorganisms due to the antimicrobial effect of essence oils. eugenol utilizados en su estudio, disminuyeron significativamente las concentraciones de AGV a las 24 horas. En el estudio de Busquet et al (25), la suplementación con dosis de 3.000 mg/l de carvacrol, brote de clavo, eugenol y aceite de orégano en forraje:concentrado en relación 50:50, disminuyó significativamente las concentraciones de AGV a las 24 horas. Castillejos et al (20) demostraron que la suplementación con dosis de $5.000 \mathrm{mg} / \mathrm{l}$ de tymol y eugenol en forraje:concentrado en relación 60:40, disminuyó significativamente las concentraciones de AGV total a las 24 horas. Petersen et al (26) observaron que el extracto vegetal Larrea disminuyó significativamente las concentraciones de AGV total en $100 \%$ heno Timothy y alfalfa:maíz en relación 50:50 a las 48 horas. Canpolat et al (27) reportaron que las suplementaciones con dosis variables de carvacrol, aceite de menta y aceite de naranja a licores ruminales colectados de carneros alimentados con hierba alfalfa:concentrado en una relación 60:40, disminuyó significativamente las concentraciones de AGV total, de ácido butírico y de ácido acético/ácido propiónico. En nuestros estudios, para todos los grupos de forraje, las suplementaciones con Timol 25, orégano 25, syzygium 200 ppm redujeron significativamente la concentración de AGV isovalérico en 24 horas, al compararlo con grupos de control positivo y negativo ( $p<0.05)$. Castillejos et al (20) observaron que suplementaciones con $500 \mathrm{mg} / \mathrm{l}$ de timol y eugenol disminuyeron significativamente las concentraciones de isovalerato e isobutirato a las 24 horas. Los hallazgos de estos investigadores apoyan los de este estudio.

De igual manera, a las 24 horas, la concentración de ácido propiónico disminuyó en grupos RMT, concentrado y heno con suplementaciones de 25 ppm de timol, al compararlo con los grupos de control positivo y negativo $(p<0.05)$. La concentración isovalérica disminuyó significativamente, especialmente con timol 25 y 200 ppm de syzygium, suplementados al grupo RMT, concentrado y heno, al compararlo con los grupos de control positivo y negativo $(p<0.05)$. Las concentraciones de ácido acético/ácido propiónico disminuyeron significativamente con 200 ppm de zingiber y syzygium 200 suplementados a grupos RMT, concentrado y heno, al compararlo con grupos de control positivo y negativo. Los resultados del estudio sobre las concentraciones AGV para grupos de forraje a las 24 horas, están de acuerdo con los encontrados por los investigadores mencionados anteriormente. La disminución de la concentración de AGV en el licor ruminal se puede atribuir a actividad reducida de microorganismos ruminales, debido al efecto antimicrobiano de los aceites esenciales. 
In the present study, Syzygium $200 \mathrm{ppm}$ supplementation increased propionic acid production at $8 \mathrm{~h}$ for hay. Propionic acid production at $24 \mathrm{~h}$ increased with Zingiber $200 \mathrm{ppm}$ and Syzgium $200 \mathrm{ppm}$ supplementations for TMR and hay, and with Zingiber 200 ppm supplementation for concentrate. Acetate:propionate proportions at $8 \mathrm{~h}$ significantly increased with Oregano 25 ppm supplementation for TMR and concentrate and with Thymol $25 \mathrm{ppm}$ supplementation for hay. The same proportion at $24 \mathrm{~h}$ significantly increased with Thymol 25 and Oregano 25 ppm supplementations for all feed groups. Busquet et al (25) reported that different doses of anethol, anise oil, carvone and tea tree oil decreased the proportion of acetate and propionate, which suggests that these compounds may not be nutritionally beneficial to dairy cattle. Demirtaş et al (28) reported that $250 \mathrm{mg}$ of rosemary (Rosmarinus officinalis L.) and sage (Salvia officinalis L.) supplementations to 50:50 roughage:concentrate rations did not have any significant effect on propionate production. However, rosemary and sage extracts were found to decrease acetate:propionate proportions compared to control fermenters without supplementations. Similarly, acetate:propionate proportions tended to decrease with increased doses of essence oils. Bodas et al. (29) demonstrated that among the essence oils tested, only Rheum nobile was found to increase propionate:acetate proportion.

In conclusion essence oils can be used as alternatives to chemical supplements used for rumen regulators. Being natural, these substances are environment-friendly. The essence oils of Thymol, Oregano, Zingiber and Syzygium used in the present study have the potential of improving rumen efficiency.

\section{Acknowledgements}

The authors are grateful to Hohenheim University (Germany) for its financial and technical support.
En el presente estudio, en el caso del heno, la suplementación con 200 ppm de syzygium incrementó la producción de ácido propiónico a las 8 horas. La producción de ácido propiónico a las 24 horas se incrementó con suplementaciones de 200 ppm de zingiber y 200 ppm de syzygium para RMT y heno, y con 200 ppm de zingiber para el concentrado. Las proporciones de acetato:propionato a la 8 horas se incrementaron significativamente con suplementaciones de $25 \mathrm{ppm}$ de orégano para RMT y concentrado y con suplementación de 25 ppm de Timol para el heno. La misma proporción a las 24 horas se incrementó significativamente con suplementaciones de $25 \mathrm{ppm}$ de Timol y 25 ppm de orégano para todos los grupos de forraje. Busquet et al (25) reportaron que diferentes dosis de anetol, aceite de anís, carvone y aceite de árbol de te, disminuyeron la proporción de acetato y propionato, lo cual sugiere que estos compuestos pueden no ser nutricionalmente beneficiosos para el ganado lechero. Demirtas et al (28) reportaron que suplementaciones con $250 \mathrm{mg}$ de romero (Rosmarinus officinalis L.) y salvia (Salvia officinalis L.) en forraje: concentrado en una relación de 50:50, no tienen ningún efecto significativo sobre la producción de propionato. Sin embargo, se encontró que al comparar en fermentadores de control, sin el uso de suplementaciones, los extractos de romero y salvia disminuyeron las proporciones de acetato/propionato. De igual manera, estas proporciones tienden a disminuir con dosis aumentadas de aceites esenciales. Bodas et al (29) demostraron que de ambos aceites esenciales ensayados, se encontró que solamente el Rheum nobile incrementó la proporción acetato/propionato.

En conclusión los aceites esenciales se pueden utilizar como alternativa a los suplementos químicos utilizados para regular el rumen. Estas sustancias, por ser naturales, son amigables con el medio ambiente. Los aceites esenciales de timol, orégano, zingiber y syzygium utilizados en el presente estudio tienen el potencial para la eficiencia del rumen.

\section{Agradecimientos}

Los autores agradecen a la Universidad de Hohenheim (Alemania) por su apoyo financiero y técnico. 


\section{REFERENCES}

1. Calsamiglia S, Busquet M, Cardoza PW, Castillejos $L$, Ferret A. Invited Review: Essential oils as modifiers of rumen microbial fermentation. J Dairy Sci 2007; 90:2580-2595.

2. Goel N, Sirohi SK, Dwivedi J. Studies on the effects of methanolic extract of Cinnamomum zeylanicum on in vitro methane inhibition and rumen fermentation patterns. J Chem Pharm Res 2011; 3(6):609-615.

3. Tekeli, A. Etlik civciv rasyonlarında doğal büyüme uyarıcı olarak bitkisel ekstraktların ve propolisin kullanım olanakları. [PhD Thesis]. Adana, Turkey: University of Çukuorova, Institute of Natural and Applied Sciences;2007.

4. Hart KJ, Yanez-Ruiz DR, Duval SM, McEwan NR, Newbold CJ. Plant extracts to manipulate rumen fermmentation. Anim Feed Sci Tech 2008; 147:8-35.

5. Hirasa K, Takemassa M. Spice science technology. Marcel Dekker Inc., New York, USA; 1998.

6. Botsoglou NA, Christaki E, Florou-Paneri $P$, Giannenas I, Papageorgiou G, Spais AB. The Effect of a mixture of herbal essential oils or a-Tocoheryl Acetate on performance parameters and oxidation of body lipid in broilers. S Afr J Anim Sci 2004; 34:52-61.

7. Jantan IB, Yassin MSM, Chin CB, Chen LL, Sim NL. Antifungal activity of the essential oils nine Zingiberaceae species. Brit Poultry Sci 2003; 41(5):392-397.

8. Mitsch $\mathrm{P}$, Zitterl-Eglseer K, Kohler B, Gabler C, Losa R, Zimpernik I. The effect of two different blends of essetial oil components on the proliferation of Clostridium perfringens in the intestines of broilers chickens. Poult Sci 2004; 83:669-675.

9. Geissler Ch, Hoffmann M, Hickeli B. Determination of volatile fatty acids by gas chromatography (in German). Arch Anim Nutr 1976; 26:123-129.

10. Hinkelmann K, Kempthorne O. Design and analysis of experiments. Volume1, John Willey \& Sons Inc., New Jersey, USA; 2008.
11. SPSS. Statistical Package for the Social Sciences [Sotfware] Version 22.0. SPSS Inc; 2013

12. Alataş MS, Umucalılar HD. Bacteria of the rumen ecosystem and their roles. Atatürk University J Vet Sci 2011; 6(1):71-83.

13. Yang MG, Manoharan K, Mickelsen $\mathrm{O}$. Nutritional contribution of volatile fatty acids from the cecum of rats. J Nutr 1970; 100:545-550.

14. Benchaar $C$, Chaves AV, Fraser GR, Wang $Y$, Beauchemin KA, McAllister TA. Effects of essential oils and their components on in vitro rumen microbial fermentation. Can J Anim Sci 2007; 413-419.

15. Beauchemin KA, McGinn SM. Methane emissions from beef cattle: effects of fumaric acid, essential oil and canola oil. J Anim Sci 2006; 84:1489-1496.

16. Benchaar C, Petit HV, Berthiaume R, Whyte TD, Chouinard PY. Effects of addition of essential oils and monensin premix on digestion, ruminal fermentation, milk production, and milk composition in dairy cows. J Dairy Sci 2006; 89:4352-4364.

17. Chaves AV, Stanford K, Gibson LL, McAllister TA, Benchaar C. Effects of carvacrol and cinnamaldehyde on intake, rumen fermentation, growth performance, and carcass characteristics of growing lambs. Anim Feed Sci Tech 2008; 145:396-408.

18. Kamalak A, Canbolat Ö, Özkan ÇÖ, Atalay A. Effect of thymol on in vitro gas production, digestibility and metabolizable energy content of alfalfa hay. Kafkas Univ J Fac Med 2011; 17(2):211-216.

19. Öztürk $H$, Demirtaş $A$, Salgırlı $Y$, Pekcan $M$, Emre B, Fidancı UR. Effects of olive leaf extract on rumen microbial fermentation in in vitro semi-continuous culture system (RUSITEC). Ankara Üniv Vet Fak Derg 2012; 59(1):17-21.

20. Castillejos L, Calsamiglia S, Ferret A. Effect of essential oil active compounds on rumen microbial fermentation and nutrient flow in in vitro systems. J Dairy Sci 2006; 89:2649-2658. 
21. Garcia V, Catala-Gregori P, Madrid J, Hernandez F, Megias MD, Andrade-Montemayor HM. Potential of carvacrol to modify in vitro rumen fermentation as compared with Monensin. Animal 2007; 1:675-680.

22. Chaves AV, Schei I, Wang Y, McAllister TA, Benchaar C. Effects of carvacrol and cinnamaldehyde on microbial fermentation when added to a barley-or corn-based diet in a continuous-culture system. Can J Anim Sci 2009; 89:97-104.

23. Sirohi SK, Chaudhary PP, Goel N. Effect of inclusion of Myristica fragrans on methane production, rumen fermentation parameters and methanogens population. Vet World 2012; 5(6):335-340.

24. Cardozo PW, Calsamiglia S, Ferret A, Kamel C. Screening for the effects of natural plant extracts at different $\mathrm{pH}$ on in vitro rumen microbial fermentation of a highconcentrate diet for beef cattle. J Anim Sci 2005; 83:2572-2579.

25. Busquet $M$, Calsamiglia S, Ferret A, Kamel C. Plant extracts affect in vitro rumen microbial fermentation. J Dairy Sci 2006; 89:761-771.
26. Petersen J, Lodge-Ivey SL, Browne-Silya J, Horvath MB. Extract from Larrea influences rumen fermentation. Proceedings, Western Section, American Society Anim Sci (ASAS) 2008; 59:367-370.

27. Canbolat O, Kalkan H, Karaman S, Filya I. The effect of essential oils on the digestibility, rumen fermentation and microbial protein production. Kafkas Univ J Fac Med 2011; 17(4):557-565.

28. Demirtaş A, Öztürk $H$, Pişkin I, Demirkıran D, Salgırlı Y, Fidancı UR, Emre B. Effects of Rosemary and Sage extracts on ruminal fermentation using the rumen simulation technique (Rusitec). J Fac Vet Med Istanbul Univ 2011; 37(2):127-134.

29. Bodas R, López S, Fernández M, GarcíaGonzález R, Wallace RJ, González JS. Phytogenic additives to decrease in vitro ruminal methanogenesis. Nutritional and Foraging Ecology of Sheep and Goats. Ciheam Options Méditerranéennes 2009; 85:279-283. 